Article

\title{
Mode Switching and Collective Behavior in Chemical Oil Droplets
}

\author{
Naoto Horibe ${ }^{1}$, Martin M. Hanczyc ${ }^{2}$ and Takashi Ikegami ${ }^{1, *}$ \\ 1 Department of General Systems Studies, University of Tokyo, Komaba, Tokyo 153, Japan; \\ E-Mail: nhoribee@08.alumni.u-tokyo.ac.jp \\ 2 Institute of Physics and Chemistry, University of Southern Denmark, Campusvej 55, \\ DK-5230 Odense M, Denmark; E-Mail: martin@ifk.sdu.dk
}

* Author to whom correspondence should be addressed; E-Mail: ikeg@sacral.c.u-tokyo.ac.jp; Tel.: +81-3-5454-6535.

Received: 17 December 2010; in revised form: 31 January 2011 / Accepted: 4 March 2011 / Published: 18 March 2011

\begin{abstract}
We have characterized several dynamic aspects of a simple chemical system capable of self-movement: An oil droplet in water system. We focused on spontaneous mode switching and collective behavior of droplets as emergent properties of the system. Droplets demonstrated spontaneous mode switching by changing speed, direction and acceleration over time, and collective behaviors of droplets resulted from such autonomous characteristics. In this paper, we quantitatively measured those characteristics to show that droplets did not act completely independently in the same system, but tend to be attracted to one another and interact with each other by adjusting their motion.
\end{abstract}

Keywords: droplet; behavior; emergence; collective behavior; protocell

PACS Codes: 47.55.D-; 47.10.-g

\section{Introduction}

When chemical potential is physically linked to movement, even in simple systems such an oil droplet in water [1-7], there is potential for emergent properties. The advantage of using simple chemical systems is that the underlying chemical and physical layers can be elucidated, shedding light 
on the mechanism of the emergent property. In the case of our oil droplet system, only five different components including water comprise the entire system that supports self-movement [8]. The oil droplet forms through self-assembly. The chemicals inside the droplet react at the oil water interface and trigger a physical instability in the droplet. Due to the distribution of chemicals, the rate of reaction, and the self-association of surfactants at the interface, the symmetry of the droplet is broken [7]. Due to the resulting fluid flow coupled with Newton's third law and hydrodynamics, the droplets are able to move.

Previously we studied how a simple oil droplet in water possessed both self-movement and a rudimentary form of chemotaxis by climbing chemical gradients [8,9]. We have argued that such 'behavior' was a result of a chemical embodiment of sensory motor coupling, forming the basis for minimal cognition in simple systems [10]. Since oil droplets modify their chemical environment and are sensitive to their own chemical signals, it is feasible that higher order collective behavior may emerge from a system of multiple droplets. Previously we reported anecdotally on two droplets exhibiting avoidance behavior or following behavior, indicating that collective behavior exists in such chemical systems [9]. In this paper we quantified both single droplet behavioral modes based on droplet size, as well as collective behavior in a two oil droplet system, as a basis for emergent properties.

\section{Results and Discussion}

\subsection{Traces and Modes of Droplet Movement}

Traces of single droplet movement in time revealed characteristic patterns depending mainly on their size (volume) and time. Figure 1 shows the traces of droplets of different sizes. For example, $1 \mu \mathrm{L}$ droplets showed circular andfluctuating behavior; $20 \mu \mathrm{L}$ droplets showed directional, circular and fluctuating behavior; $50 \mu \mathrm{L}$ droplets showed vibrational, circular and fluctuating behavior.

Figure 1. Paths of droplets. The red lines indicate the paths of single droplets of varying volume $(1 \mu \mathrm{L}, 20 \mu \mathrm{L}$ and $50 \mu \mathrm{L})$ traced over the entire lifetime of an experiment.
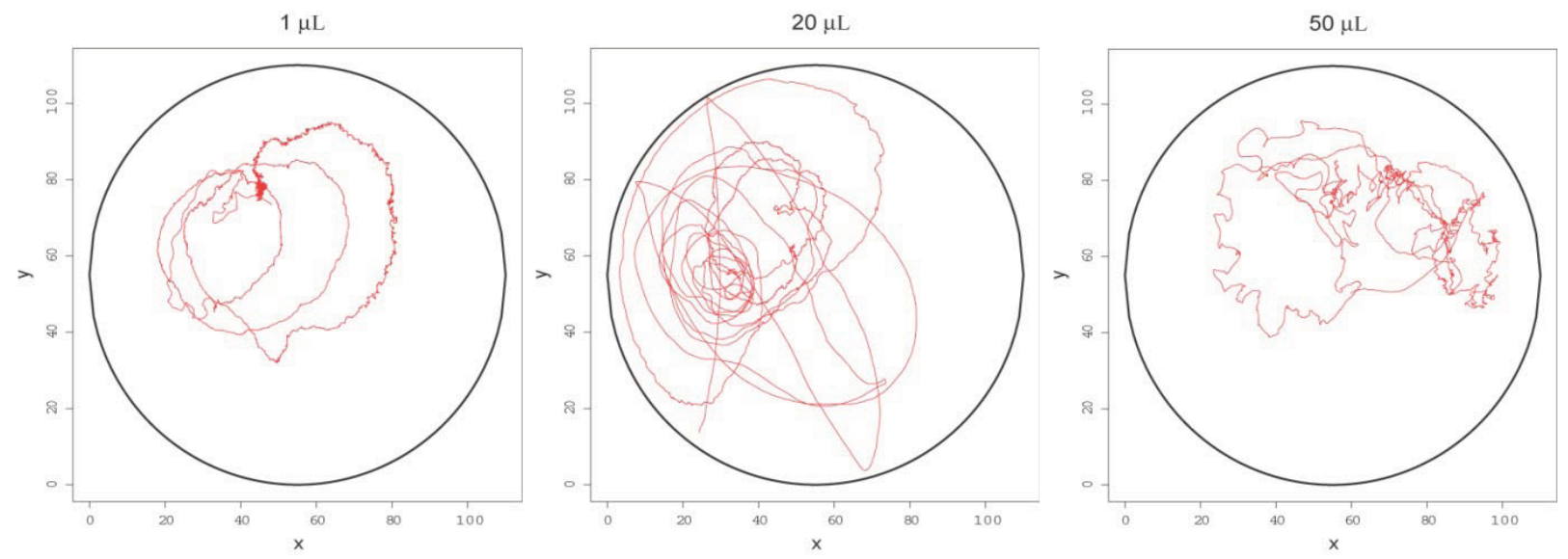

As shown in Figure 2, droplets showed complex paths consisting of at least four behavioral modes, namely directional, vibrational, fluctuating and circ ular modes. Because the reaction occurs at the surface of the droplet, the rate of reaction is largely determined by the size of droplet, with stronger reaction in larger droplets. In addition, the typically spherical droplet shape can often be distorted by flow structures occurring inside the oil droplet. The droplet shape and convectionflow of the largest 
droplets tested ( $50 \mu \mathrm{L}$ volume) became unstable and the droplets showed vibrational mode. Over time both the oil volume decreased and the reaction became weaker, and concurrently the droplets made a transition from one mode to another (e.g., vibrating mode to circular mode). In the directional mode, both droplet shape and convection flow were stable and the rate of chemical reaction was strong. When the reaction became much weaker, convection flow became unstable and this instability correlated with fluctuating behavior. More detailed analyses of mode identification and mode switching are presented below in Section 2.4 .

Figure 2. Four major behavioral modes identified and corresponding droplet shape.
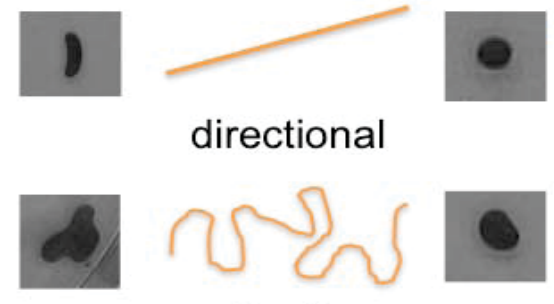

directional

fluctuating
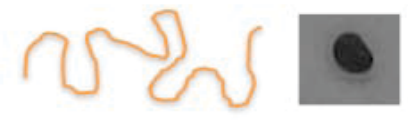

vibrating

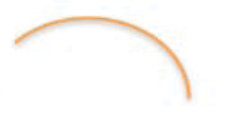

circular

\subsection{Spontaneous Change in Direction and Velocity}

Once a droplet started moving, it often stopped and then started to move again. We characterized this apparently spontaneous change in direction and velocity of moving droplets by quantifying the both the turning angle and velocity of the droplet (Figure 3). The data showed a decrease in velocity and increase in turning angle over time as the droplet aged. As also shown in Figure 3, turning angle was negatively correlated with velocity. This suggested that the droplets often changed their direction while stopped. This applied to all droplet sizes tested. Droplets showed fluctuating mode in the stop duration, and we found from numerical simulation that this mode has an unstable axis of convection flow resulting in spontaneous changes in the direction of movement [7]. This was also corroborated with microscopic observation of flow structures in the oil droplets (data not shown).

Previously we reported on how droplets react to chemical gradients [8]. Here we tested how droplets react to a collision with an immovable physical object. We calculated the coefficient of restitution (COR) of droplets placed into the dish with a glass triangle providing a flat surface for the droplets to collide with, as shown in Figure 4A. We defined COR as $\|v(t+1)\| / \| v t||$.The COR indicates elasticity by comparing the velocities of objects before and after an event, such as a collision. For comparison, we calculated the density distribution of the COR for droplets not undergoing collisions (Figure 4B). This data showed spontaneous production or consumption of kinetic energy with mean values around 1 . The chemical reaction on the surface could be converted to kinetic energy possibly through shape change and/or fluid dynamics where COR values were $>1$. A COR larger than 1 indicates that the chemical reaction was re-activated or enhanced. However when droplets underwent collisions, then distribution changed as shown in Figure 4C, with generally lower COR and even a few values indicating a perfectly inelastic system. In this case, kinetic energy could be consumed to distort the droplet shape during the collision. 
Figure 3. Correlation between velocity and turning angle showing a strong negative correlation. Data are shown for $20 \mu \mathrm{L}$ droplets only.
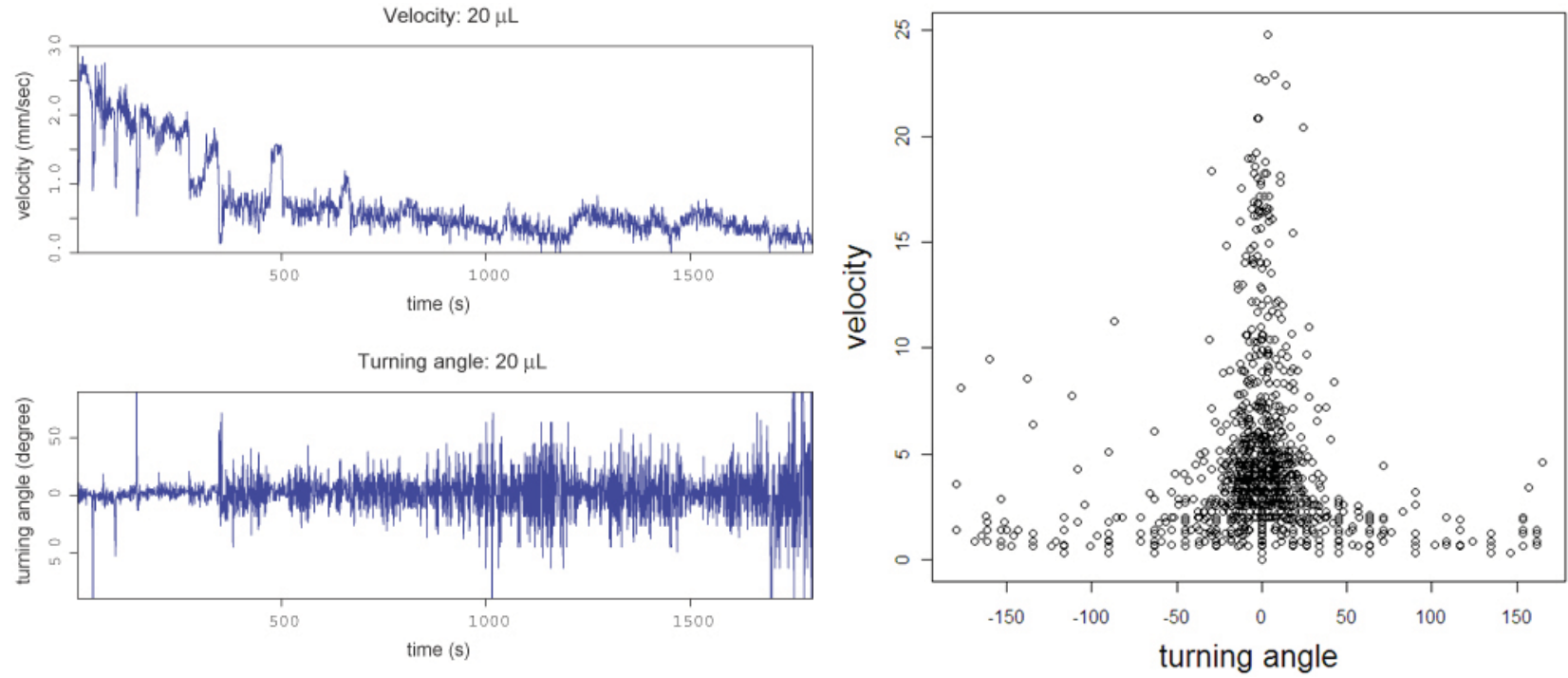

Figure 4. Coefficient of restitution measurements. (A). The droplets were tested in a glass dish of $60 \mathrm{~mm}$ diameter with glass separators of $48 \mathrm{~mm}$ length to serve as flat walls; (B). The coefficients for all droplets tested where there was no collision but spontaneous change in velocity; (C). The coefficients for all droplets tested where there was a collision with a wall; (D). The coefficients according to droplet size (in $\mu \mathrm{L}$ ).
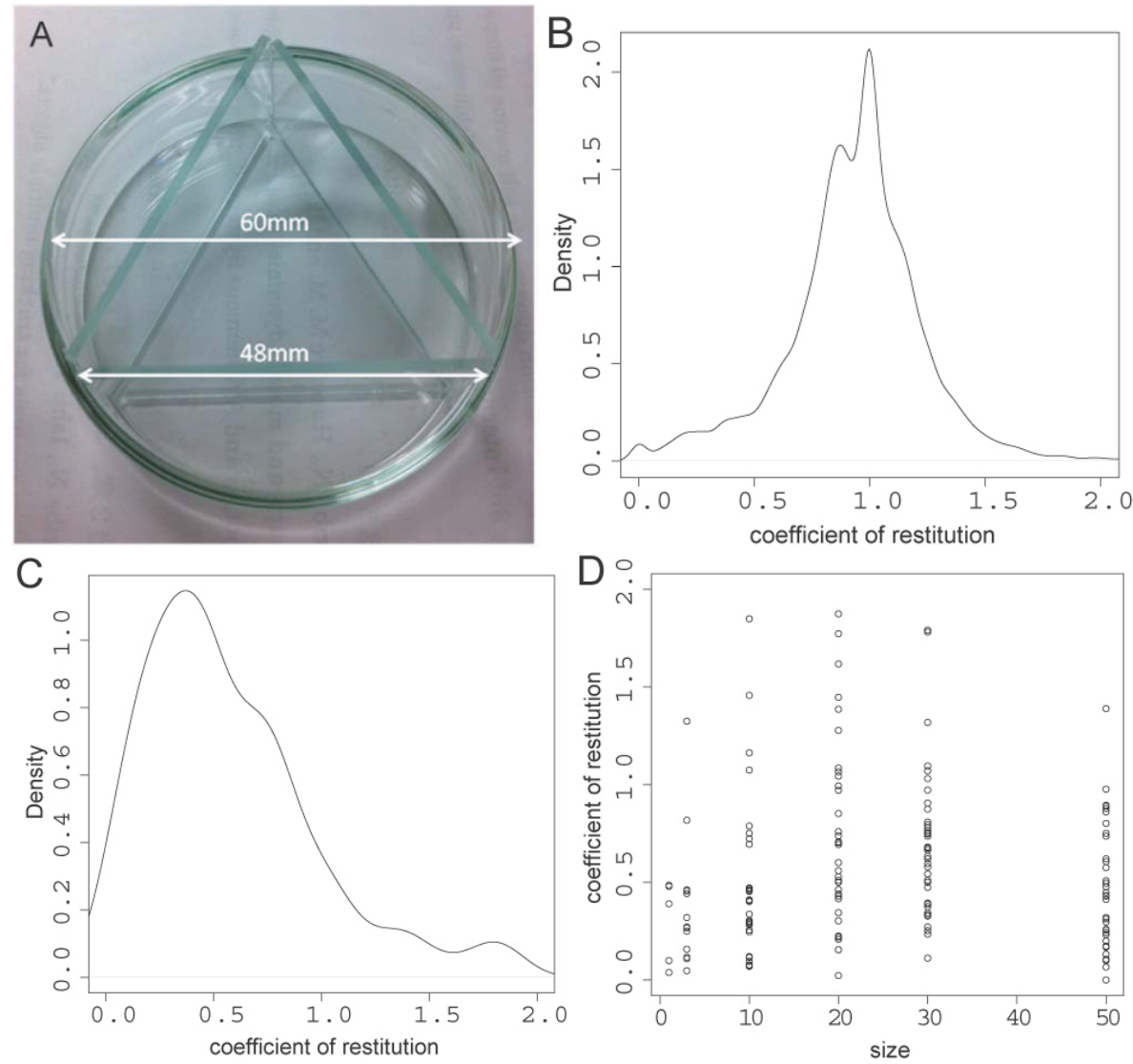


\subsection{Size Dependent Change of Coefficient of Restitution}

When we plotted the COR against the size of the droplets, we saw size dependent change of the coefficient (Figure 4D). The smaller $(1,3,10 \mu \mathrm{L})$ and larger droplets $(50 \mu \mathrm{L})$ showed smaller COR values. When the $50 \mu \mathrm{L}$ droplets collided with a wall, the shape of the droplets was easily distorted. Therefore, the smaller COR at this size was due to the transmission of energy from kinetic to surface energy. In smaller droplets $(1,3 \mu \mathrm{L})$, they were more highly bound to a spherical shape due to the Laplace pressure. In the small droplets $(1-10 \mu \mathrm{L})$, the small COR may be due to the thermal dissipation or disappearance of the internal convectiofow. Droplets showed wide range of COR depending on the state of the system. Therefore the system state may affect not only the interaction of a droplet with a wall but also droplet-droplet interactions.

\subsection{Behavioral Modes and Their Transitions in Relation to Size}

We used a self-organizing map (SOM) and identified behavioral modes using a U-matrix [11]. The standard algorithm for the $S O M$ is as follows. We defined the data set $S=\left\{X_{1}, X_{2}, X_{3}, \ldots, X_{N}\right\}$ where $\mathrm{X}_{\mathrm{k}}$ is a $\mathrm{n}$-dimensional vector $\left(\mathrm{x}_{1}, \mathrm{x}_{2}, \mathrm{x}_{3}, \ldots, \mathrm{x}_{\mathrm{n}}\right)$ and nodes on a SOM, which are connected with the neighboring nodes, $\mathrm{W}_{\mathrm{i}, \mathrm{j}}$ where $\mathrm{i}, \mathrm{j}$ indicate the position of the node on the SOM having a weight vector with the same dimension as the data vector $\left(\mathrm{w}_{1}, \mathrm{w}_{2}, \mathrm{w}_{3}, \ldots, \mathrm{w}_{\mathrm{n}}\right)$. One data vector from the data set was randomly chosen and the node that satisfied $\min _{\mathrm{i}, \mathrm{j}}\left|\mathrm{X}_{\mathrm{k}}-\mathrm{W}_{\mathrm{i}, \mathrm{j}}\right|$ was determined. Then the weight for the node $\mathrm{W}_{\mathrm{i}, \mathrm{j}}$ and the neighboring nodes were updated with a certain learning rate $\epsilon$ (the node) and $\eta$ (the neighboring node, $\epsilon>\eta$ ). Iterating this process, we obtained a self-organized network where the neighboring nodes had similar weights (Figure 5). Finishing the update of the weights on the nodes, we calculated the distance between nodes and then obtained a U-matrix containing information on the distance between nodes $\left(U(i, j)=\left|W_{n, m}-W_{n, m-1}\right|\right)$. Finally, we divided SOM into several parts containing similar nodes using the U-matrix.

Figure 5. The standard algorithm of SOM is illustrated. Each color represents different weights on each node. Weights are randomly attributed at first (left) and modified through unsupervised learning process. Finishing the learning (center) of SOM, nodes with similar weights make clusters and they can be divided into several parts by using the U-matrix (right).
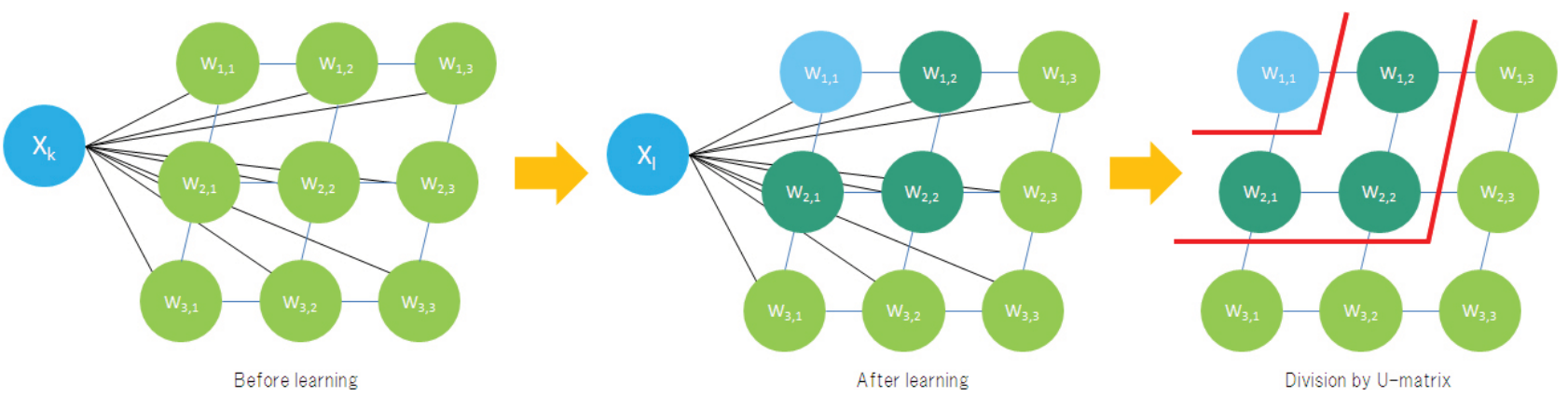

The velocity and turning angle of the droplets were recorded every second, binned into 20 -second intervals, and then normalized. The data was used for unsupervised learning of SOM. The SOM was 
then divided into several parts using the U-matrix and data mapped on it. We used a threshold for the U-matrix so that the SOM would identify only main clusters.

Four types of main modes were identified (Figure 6). They were fluctuating, circular, directional and vibrating mode. Small velocity and large turning angle were observed in fluctuating mode. On the other hand, large velocity and small turning angle were observed in directional mode. Circular and vibrating mode showed intermediate velocity and turning angle but they were unstable in vibrating mode. In smaller droplets ( $1 \mu \mathrm{L}$ volume), velocity was relatively unstable compared to larger droplets $(50 \mu \mathrm{L})$. Such behaviors were identified as the other modes on the SOM. However, they were partly directional and circular mode, so we named those mixed modes as fluctuating directional mode (III') and fluctuating circular mode (II').

Figure 6. Identification of the behavioral modes using SOM. Each polygon in the circle represents the weights for the node, where the upper half shows velocity and the lower half shows turning angle. Red lines define the borders of the each behavioral mode, which are determined from U-matrix, and four behavioral modes are identified: fluctuating mode (I), circular mode (II), directional mode (III) and vibrating mode (IV), along with two mixed modes: fluctuating directional mode (III') and fluctuating circular mode (II'). The dots on the SOM represent motion of the droplet in 20 seconds intervals and the colors represent sizes of droplets: blue for $1 \mu \mathrm{L}$, yellow for $20 \mu \mathrm{L}$ and red for $50 \mu \mathrm{L}$.

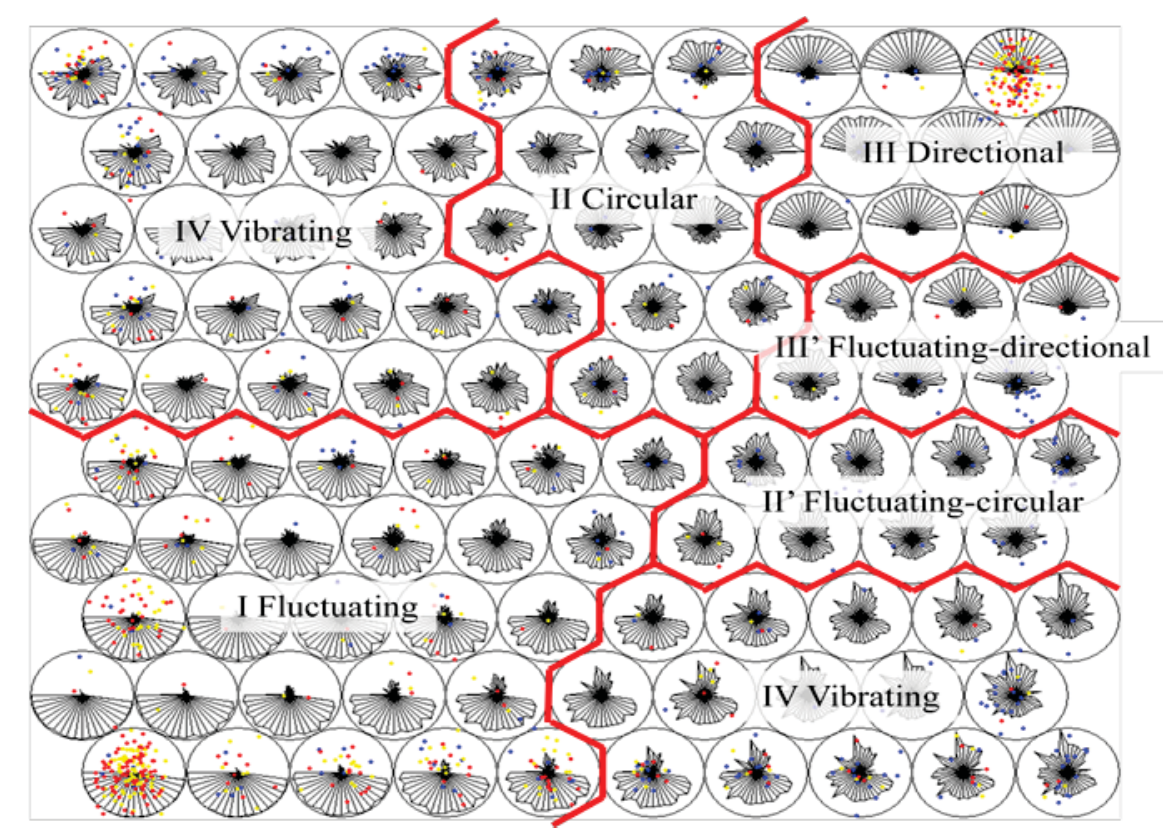

The mixture percentiles of behavioral modes were calculated from mapping on SOM and transition probability and transition pattern were calculated from SOM (Figure 7). The components of the paths were different depending on the droplet size. Small droplets $(<10 \mu \mathrm{L})$ mainly showed directional and fluctuating mode and the duration for staying in the directional mode became shorter as time passed. Middle-sized droplets $(10-30 \mu \mathrm{L})$ showed directional mode in its former stage and switching between circular and fluctuating mode in its later stage. Both small $(1 \mu \mathrm{L})$ and middle size $(20 \mu \mathrm{L})$ droplets did not show vibrating mode. Large droplets $(50 \mu \mathrm{L})$ had a tendency to stay in vibrating mode but occasionally switched to the other three modes. 


\subsection{Collective Behavior}

To see the relation between behavioral mode and collective behavior in droplets, we placed two droplets in one glass plate and recorded their paths for $60 \mathrm{~min}$. As a control, we superimposed the trails of two single droplets of the same size selected at random from discrete plates. We conducted the experiment for three different sizes of the droplets: 3,20 and $50 \mu \mathrm{L}$, because their dominant behavioral modes were completely different from one another (Figures 6 and 7). The result for the first 20 min of movement for $20 \mu \mathrm{L}$ droplets is shown in Figure 8A. We found that the distance between the droplets was smaller when the droplets were in the same plate. This is evidence of an attracting force. The attracting force became weaker over time (Figures $8 \mathrm{~B}$ and $7 \mathrm{C}$ ). Larger droplets $(50 \mu \mathrm{L})$ did not show collective behavior even in the first $20 \mathrm{~min}$ (data not shown). The $3 \mu \mathrm{L}$ droplets showed an attracting behavior similar to the $20 \mu \mathrm{L}$ droplets that dissipated over time. We noted that convection flow was weak in older droplets and unstable in the larger droplets, which may account for their inability to show attracting behavior. Droplets that sustained strong organized convection flow also displayed attracting behavior.

Figure 7. Transition probabilities between four behavioral modes for droplets of different volumes. (A). $1 \mu \mathrm{L}$ droplet; (B). $20 \mu \mathrm{L}$ droplet; (C). $50 \mu \mathrm{L}$ droplet.

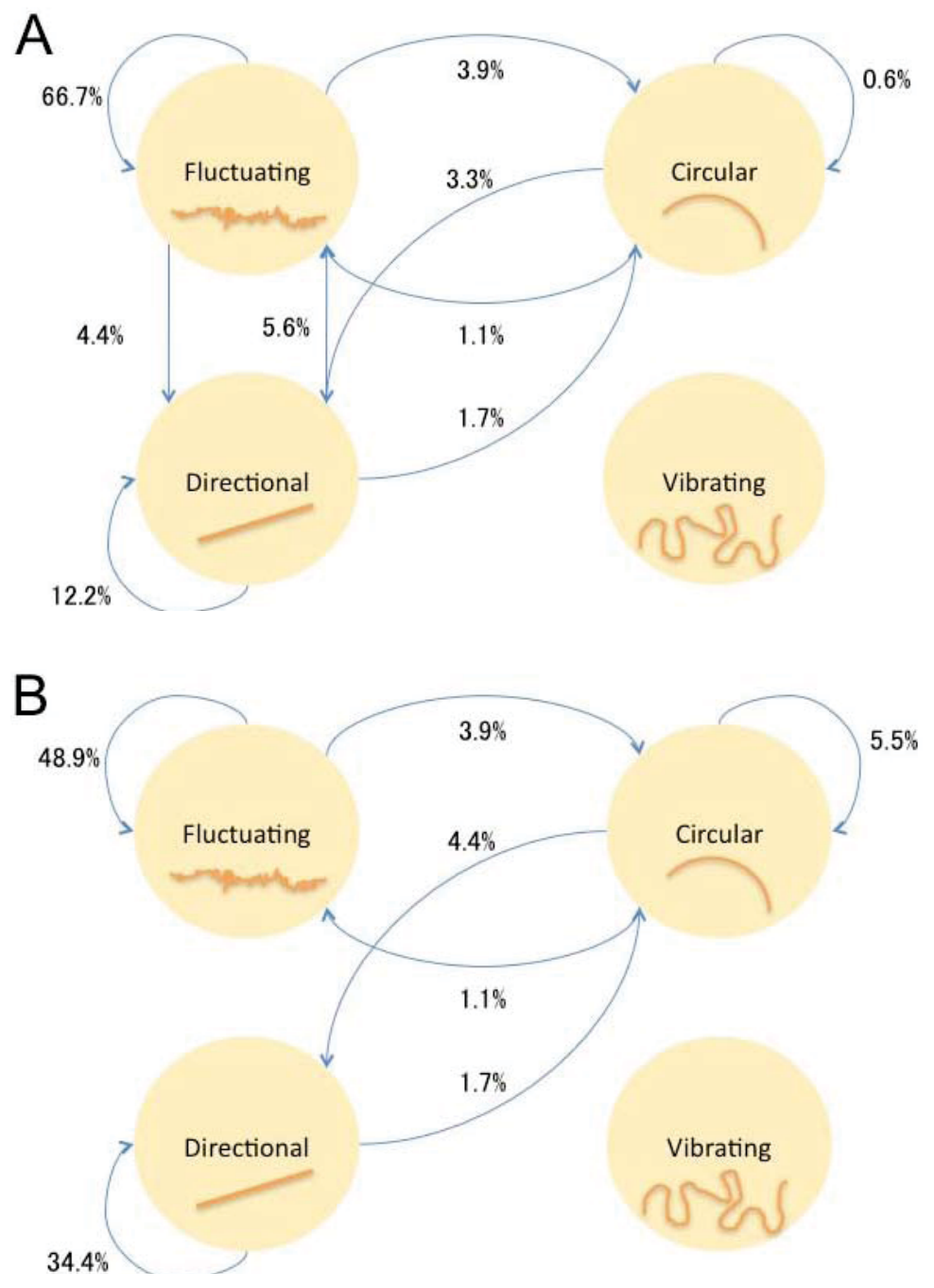


Figure 7. Cont.

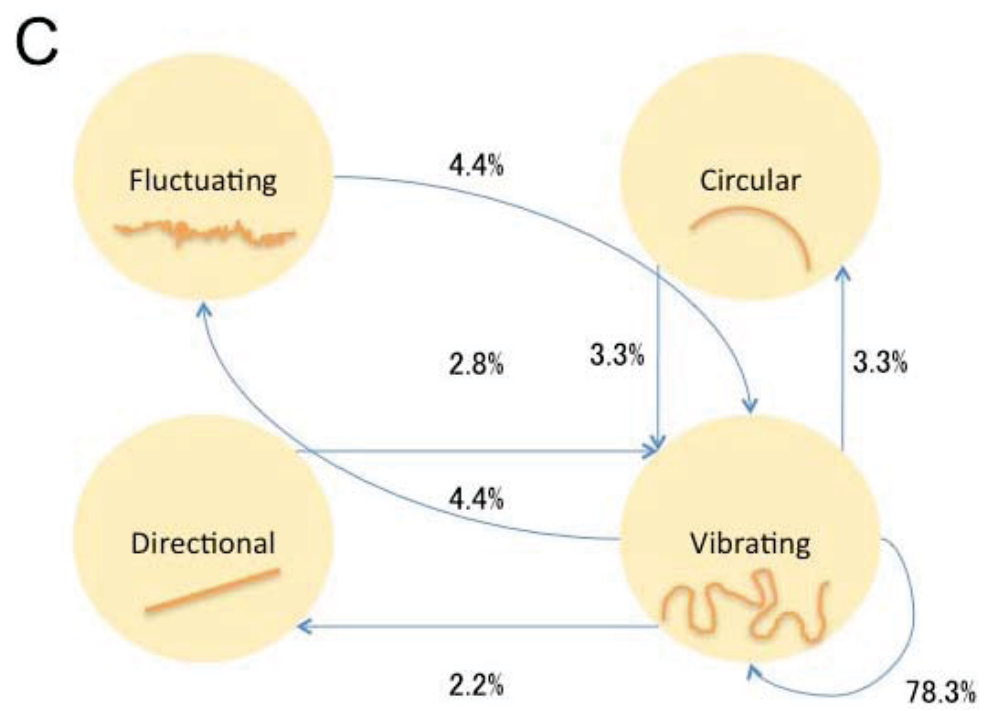

Figure 8. Mutual distance measurements between two droplets in two discrete plates (control) or in the same plate. (A). Mutual distance between two droplets of the size $20 \mu \mathrm{L}$ in the first $20 \mathrm{~min}$ if the experiment; (B). The next $20 \mathrm{~min}$ and (C). the final $20 \mathrm{~min}$.
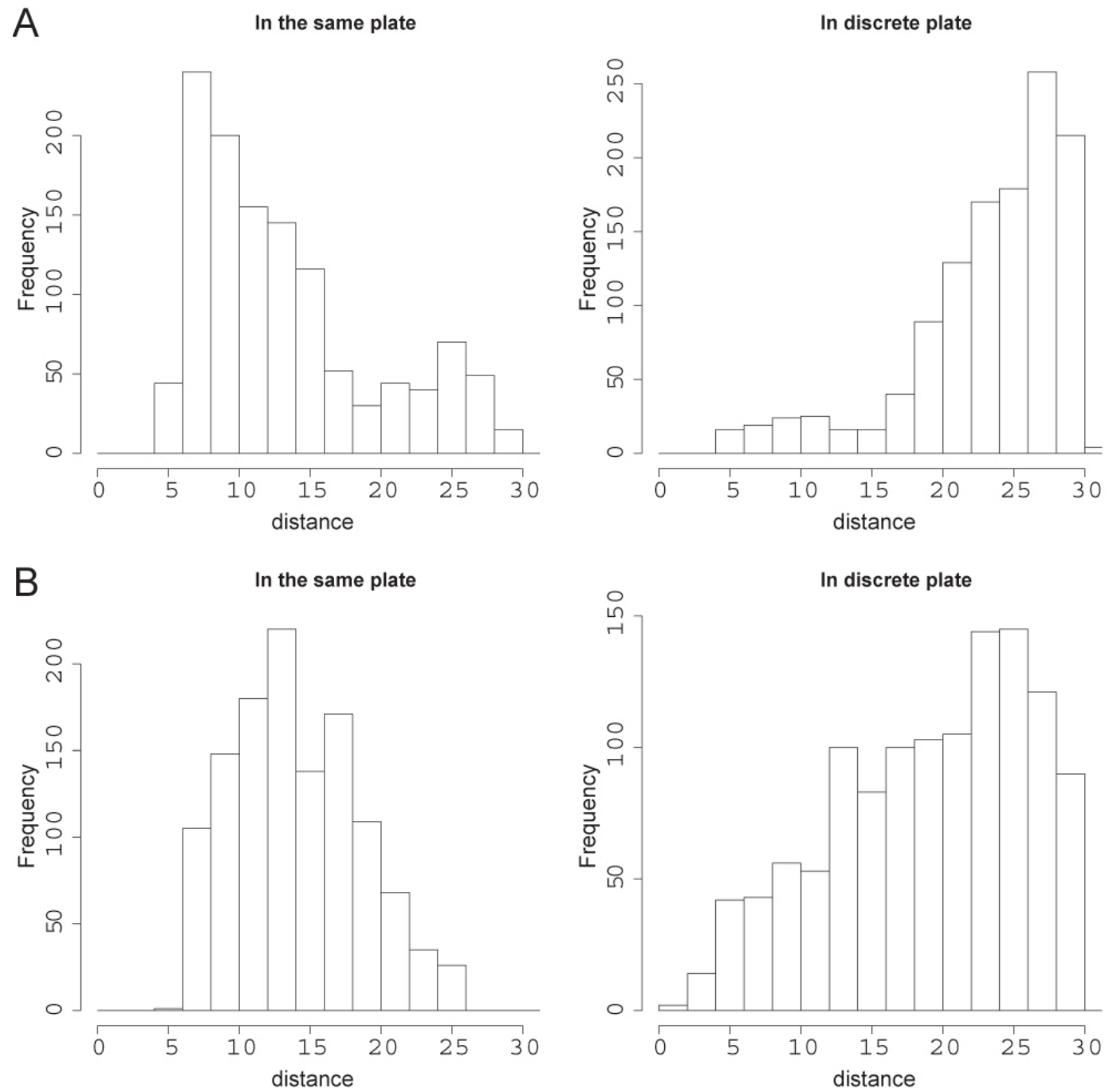
Figure 8. Cont.
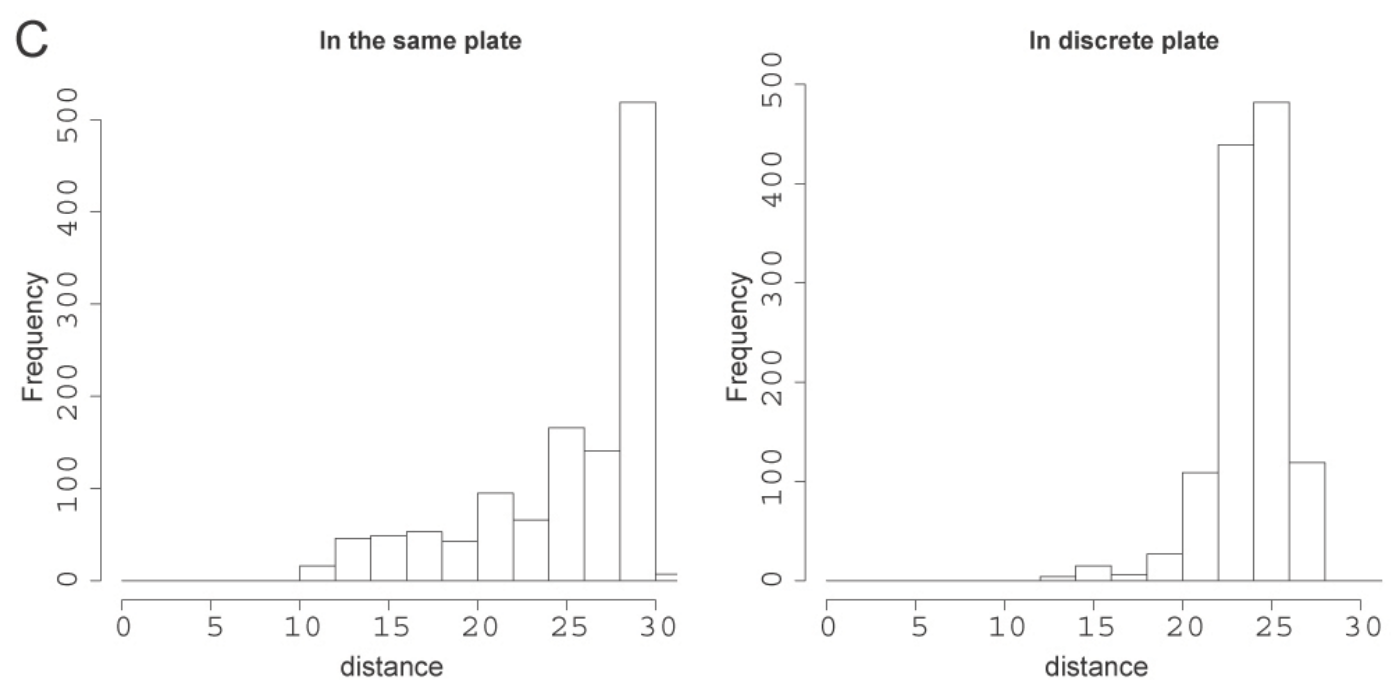

\subsection{Droplet Size and Behavior}

The size of the droplet is responsible for its shape, convectionflow and reaction rate, and these together influenced the behavioral mode as well and transitions between behavioral modes. This also influenced how a droplet physically interacted with an inanimate wall or another animate droplet.

Here we analyzed the movement or behavior of single droplets and noted that there were several types of motion for this system, depending on both size and age of the droplet. This was most apparent when mapping out the different modes of behavior for droplets of different size along with the intermodal transition frequencies (Figure 7). It was also noted that the behavior of a droplet was not robust but shows fluctuation. This was most apparent in the COR data (Figure 4) where both the smallest $(1-10 \mu \mathrm{L})$ and largest $(50 \mu \mathrm{L})$ droplets tested exhibited non-elastic properties. But this was not strictly observed with at least a few droplets from each size class exhibiting elastic behavior. Therefore a droplet from each size class may exhibit different modes of behavior and interaction depending on factors that affect the state of the droplet. For example, we have reported elsewhere that the behavioral mode transition probabilities were also affected by the previous mode due to a memory effect [12].

We also characterized the behavior of two droplets moving in the same system and show that there was an attracting effect where two droplets tended to cluster together in space. We hypothesize that this attraction was due to droplet hydrodynamics influenced by local chemical signals produced by the droplets. The source of the attraction force may come from reciprocal enforcement of convection flows. Because convection flow in some sizes of droplets was unstable, they could not reinforce their flows and therefore they did not exhibit this attractive force (for example, large $50 \mu \mathrm{L}$ droplets). It was clear from previous studies that single droplets produced chemical gradients and that single droplets sensed and responded chemotactically to such gradients [8]. Here we showed the ability to sense and respond resulted in higher order collective behavior from such a simple chemical system. 


\section{Experimental}

Nitrobenzene was purchased from Kanto Chemical Co., Ltd and oleic anhydride, oleic acid, and sodium hydroxide were purchased from Tokyo Chemical Industry Co., Ltd. Glass dishes of 35, 60 and $110 \mathrm{~mm}$ diameter were purchased from AS ONE Corporation.

Fresh oleic anhydride was mixed with nitrobenzene at a ratio of $1: 1 \mathrm{v} / \mathrm{v}$. The oil was then added to a dish containing $10 \mathrm{mM}$ oleate micelles at $\mathrm{pH} \mathrm{11.} \mathrm{The} \mathrm{movement} \mathrm{of} \mathrm{the} \mathrm{droplets} \mathrm{in} \mathrm{the} \mathrm{dish} \mathrm{was} \mathrm{then}$ recorded in real time using a digital video camera (HDR-HC1, SONY, Japan). Running droplets were analyzed in real time using an open software called R [13] and open-cv software (version 1.1.0) [14].

To monitor behavioral modes, $20 \mathrm{~mL}$ of $10 \mathrm{mM}$ oleate micelles at $\mathrm{pH} 11$ were added to the $100 \mathrm{~mm}$ glass dish. Droplets of $1,3,10,20,30$, and $50 \mu \mathrm{L}$ were added and paths recorded over time.

For coefficient of restitution, $5 \mathrm{~mL}$ of oleate micelles were added to the $60 \mathrm{~mm}$ glass dish containing a triangle glass wall (Figure 4A). Droplets of 1,3,10, 20, 30 and $50 \mu \mathrm{L}$ were then added and paths recorded over time.

For collective behavior, $2 \mathrm{~mL}$ of oleate micelles were added to the $35 \mathrm{~mm}$ dish with a $27 \mathrm{~mm}$ glass base. Droplets pairs of $3 \mu \mathrm{L}$ and $3 \mu \mathrm{L}, 20 \mu \mathrm{L}$ and $20 \mu \mathrm{L}$, and $50 \mu \mathrm{L}$ and $50 \mu \mathrm{L}$ were then added and their paths recorded over time.

\section{Conclusion}

The behavior of a protocell in the form of an oil droplet, that possesses a body and metabolism (but no DNA or ability to replicate), was recorded and analyzed while changing only one parameter: body size. By manipulating this one parameter over a few orders of magnitude, we observed differences in behavior (motion), body shape, as well as potential for higher order interactions among protocells. We also found that spontaneous events showed a COR $>1$ more often than the case where the droplet collided with a wall. We interpret this spontaneous activation, coming from both the internal state of the droplet as well as its history imprinted on its self-modified chemical environment, as the basis for autonomy. Certain properties such as autonomy and collective behavior were emergent in this system, and the parameters (such as stable convection flow and collective behavior) that support such emergent properties were ascertained.

Although the behavior of such simple non-living systems is basic, it is also not easily predictable or deterministic. Therefore we study such systems as models of autonomy and minimal cognition based on physico-chemical principles [10]. Through such systematic exploration as presented here and in our ongoing research we explore how both behaviors, intermodal transitions, and internal states relate to the propensity of non-living simple systems to possess the basis of minimal cognition as an emergent property of physico-chemical systems.

\section{Acknowledgements}

We thank our collaborators Tadashi Sugawara, Kentaro Suzuki and Taro Toyota for helpful discussions and development of the oil droplet systems. The experiments were started when Martin Hanczyc was staying at the University of Tokyo as a visiting professor. This work has been partially supported by a Grant-in-Aid for Scientific Research on Priority Areas "Emergence of Adaptive Motor 
Function through Interaction between Body, Brain and Environment" from the Japanese Ministry of Education, Culture, Sports, Science and Technology.

\section{References}

1. Mangome, N.; Yoshikawa, K. Nonlinear oscillation and ameba-like motion in an oil/water system. J. Phys. Chem. 1996, 100, 19102-19105.

2. Dos Santos, F.; Ondarcuhu T. Free-running droplets. Phys. Rev. Lett. 1995, 75, 2972-2975.

3. Sumino, Y.; Kitahata, H.; Yoshikawa, K.; Nagayama, M.; Nomura, S.-i.M.; Magome, N.; Mori, Y. Chemosensitive running droplet. Phys. Rev. E 2005, 72, 041603.

4. Lee, S.-W.; Kwok, D.; Laibinis, P. Chemical influences on adsorption-mediated self-propelled drop movement. Phys. Rev. E 2002, 65, 051602.

5. Sumino, Y.; Magome, N.; Hamada, T.; Yoshikawa, K. Self-running droplet: Emergence of regular motion from nonequilibrium noise. Phys. Rev. Lett. 2005, 94, 068301.

6. Rybalko, S.; Magome, N.; Yoshikawa, K. Forward and backward laser-guided motion of an oil droplet. Phys. Rev. E 2004, 70, 046301.

7. Matsuno, H.; Hanczyc, M.M.; Ikegami, T. Self-maintained movements of droplets with convection flow. In Proceedings of the 3rd Australian Conference on Progress in Artificial Life, Springer-Verlag Berlin: Heidelberg, Germany, 2007; pp. 179-188.

8. Hanczyc, M.M.; Toyota, T.; Ikegami, T.; Packard, N.; Sugawara, T. Fatty acid chemistry at the oil-water interface: Self-propelled oil droplets. J. Am. Chem. Soc. 2007, 129, 9386-9391.

9. Toyota, T.; Maru, N.; Hanczyc, M.M.; Ikegami, T.; Sugawara, T. Self-propelled oil droplets consuming "Fuel” surfactant. J. Am. Chem. Soc. 2009, 131, 5012-5013.

10. Hanczyc, M.M.; Ikegami, T. Chemical basis for minimal cognition. Artif. Life 2010, 16, 233-243.

11. Kohonen, T. Self-Organizing Maps, 3rd ed.; Springer: Berlin, Germany, 2000; p. 521.

12. Horibe, N.; Hanczyc, M.M.; Ikegami, T. Shape and motion dynamics in self-moving oil droplets. Robot. Auton. Systems 2011, submitted.

13. $R$, Version 2.7.2. Available online: http://www.r-project.org/ (accessed on 25 August 2008).

14. Open $C V$, Version 1.1.0. Available online: http://opencv.willowgarage.com/wiki/ (accessed on 8 September 2009).

(C) 2011 by the authors; licensee MDPI, Basel, Switzerland. This article is an open access article distributed under the terms and conditions of the Creative Commons Attribution license (http://creativecommons.org/licenses/by/3.0/). 